\title{
Poles in $K^{-} \pi^{+}$Amplitude
}

\section{P. C. Magalhães*}

Instituto de Física, Universidade de São Paulo, C.P. 66318, 05315-970, São Paulo, SP, Brazil.

E-mail: patricialif.usp.br

\section{R. Robilotta}

Instituto de Física, Universidade de São Paulo, C.P. 66318, 05315-970, São Paulo, SP, Brazil.

E-mail: robilotta@if.usp.br

We present a simple chiral model for the $J=0, I=1 / 2$, elastic $K \pi$ amplitude which allows a transparent determination of its poles and preserve the essential physics. In the case of the $K$ matrix approximation, the model yields a quadratic equation in $s$. The solutions to this equation can then be well approximated by polynomials of masses and coupling constants. This analytic structure allows a clear understanding why, depending on the values of one of the coupling constants, one may have one or two physical poles. The model yields a pole, associated with the $\kappa$, at $\sqrt{s}=(0.75-i 0.24) \mathrm{GeV}$.

8th Conference Quark Confinement and the Hadron Spectrum September 1-62008

Mainz, Germany

\footnotetext{
* Speaker.
} 
The $K^{-} \pi^{+}$elastic scaterring amplitude for $(J, I)=(1 / 2,0)$ is discribed by the diagram

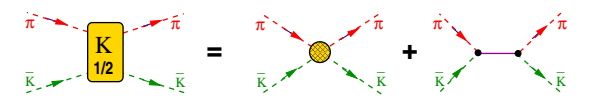

The contact[1] and resonant[2] term are derived from $S U(3) \times S U(3)$ chiral effective lagrangians

$$
\mathscr{L}^{(2)}=\frac{F^{2}}{4}\left\langle\nabla_{\mu} U^{\dagger} \nabla^{\mu} U+\chi^{\dagger} U+\chi U^{\dagger}\right\rangle+c_{d}\left\langle S u_{\mu} u^{\mu}\right\rangle+c_{m}\left\langle S \chi_{+}\right\rangle .
$$

$U$ is the pseudoscalar field, $S$ represent scalar resonaces and $c_{d}$ and $c_{m}$ are scalar-pseudoscalar coupling constants.

The $(J, I)=(0,1 / 2)$ amplitude is unitarized considering all $K \pi$ buble loop interactions[3]

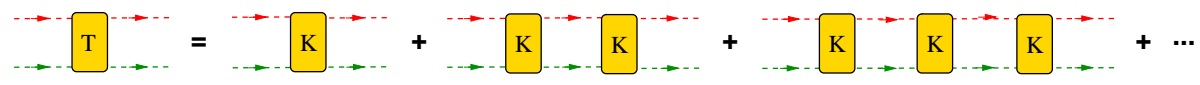

and the amplitude is written as

$$
\begin{aligned}
& T_{1 / 2}(s)=\gamma^{2}(s) / D(s), \\
& D(s)=\left[m_{R}^{2}-s+\gamma^{2}(s) \bar{R}_{1 / 2}(s)\right]-i\left[\gamma^{2}(s) \frac{\rho(s)}{16 \pi}\right],
\end{aligned}
$$

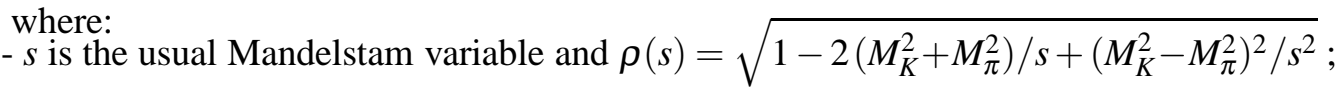

- $m_{R}$ is the parameter present in the chiral lagrangian, called nominal resonance mass;

- $\bar{R}_{1 / 2}(s)$ is the function describing off-shell effects in the two-meson propagator, given by

$$
\begin{aligned}
\bar{R}_{1 / 2}(s) & =-\mathfrak{R}\left[L(s)-L\left(m_{R}^{2}\right)\right] / 16 \pi^{2}, \\
\Re L(s) & \left.=\rho(s) \log [(1-\sigma) /(1+\sigma)]-2+\left[\left(M_{K}^{2}-M_{\pi}^{2}\right) / s\right] \log \left(M_{K} / M_{\pi}\right)\right], \\
\sigma & =\sqrt{\left|s-\left(M_{K}+M_{\pi}\right)^{2}\right| /\left|s-\left(M_{K}-M_{\pi}\right)^{2}\right|} ;
\end{aligned}
$$

- $\bar{R}_{1 / 2}\left(m_{R}^{2}\right)=0$ by construction and therefore the phase shift is $\pi / 2$ at $s=m_{R}^{2}$;

- $\gamma^{2}(s)$ is the function which incorporates chiral dynamics, given by

$$
\begin{aligned}
\gamma^{2}(s)=\left\{\left(1 / F^{2}\right)\right. & {\left.\left[\left(1-3 \rho^{2}(s) / 8\right) s-\left(M_{\pi}^{2}+M_{K}^{2}\right)\right]\left(m_{R}^{2}-s\right)\right\}_{L} } \\
+ & \left\{\left(3 / F^{4}\right)\left[c_{d}\left(s-M_{\pi}^{2}-M_{K}^{2}\right)+c_{m}\left(4 M_{K}^{2}+5 M_{\pi}^{2}\right) / 6\right]^{2}\right\}_{R} .
\end{aligned}
$$

Poles are zeros in $D(s)$ (2). In the results from numerical solution is dificult to identify dynamic. In other way, analitical solution are approximation but transparent physics. To find the anlitical equation we consider $m_{\pi}=0 \Rightarrow S U(2)$ limit and K-matrix approximation $\rightarrow \bar{R}_{1 / 2}(s)=0$. Then $D(s)$ became a quartic function

$$
\begin{aligned}
& \left(\frac{5}{8}-\frac{3 c_{d}^{2}}{8}\right) s^{4}+\left[-\left(5 m_{r}^{2}+7 m_{K}^{2}\right) / 8+\frac{c_{d}}{F^{2}}\left(9 c_{d}-4 c_{m}\right) m_{K}^{2}+i 16 \pi F^{2}\right] s^{3} \\
& +\left[\left(7 m_{r}^{2}-m_{K}^{2}\right) \frac{m_{K}^{2}}{8}-\left(c_{d}-2 c_{m} / 3\right)\left(9 c_{d}-2 c_{m}\right) \frac{M_{K}^{4}}{F^{2}} m_{r}^{2}-i 16 \pi F^{2}\right] s^{2} \\
& +\left[\left(m_{r}^{2}+3 m_{K}^{2}\right) / 8+3\left(c_{d}-2 c_{m} / 3\right)^{2} \frac{m_{K}^{2}}{F^{2}}\right] M_{K}^{4} s-3 m_{r}^{2} M_{K}^{6} / 8=0
\end{aligned}
$$

Close to pole position $m_{K}^{2} /|s| \ll 1$ and $D(s)$ is reduced to a quadratic function

$$
\begin{aligned}
& A s^{2}+B s+C=0 \quad A=\left[5 / 8-3 c_{d}^{2} / F^{2}\right] ; \\
& B=\left[-\left(5 m_{R}^{2}+7 M_{K}^{2}\right) / 8+c_{d}\left(9 c_{d}-4 c_{m}\right) \frac{M_{K}^{2}}{F^{2}}+i 16 \pi F^{2}\right] ; \\
& C=\left[7 M_{K}^{2} / 8-i 16 \pi F^{2}\right] m_{R}^{2} \text {. }
\end{aligned}
$$


The coefficient $A=5 / 8-3 c_{d}^{2} / F^{2}$ is very important. $A=0 \rightarrow c_{d} / F=\sqrt{5 / 24}=0.047$ and the quadratic function have a single solution

$$
s_{-}(0)=\frac{\left[7 M_{K}^{2} / 5-i 128 \pi F^{2} / 5\right]}{1+\left[\frac{7 M_{K}^{2}}{5}-8 c_{d}\left(9 c_{d}-4 c_{m}\right) \frac{M_{K}^{2}}{F^{2}}-i 128 \pi \frac{F^{2}}{5}\right] / m_{R}^{2}} .
$$

In $A=5 / 8 \rightarrow c_{d}=0$ resonance $R$ is decoupled bound state in the real axis,

$$
s_{+}(5 / 8)=m_{R}^{2} \quad \text { and } \quad s_{-}(5 / 8)=\left[7 M_{K}^{2} / 5-i 128 \pi F^{2} / 5\right]
$$

Analitical solution: (approximate),

$$
\begin{aligned}
s_{+}= & \frac{1}{A}\left\{\frac{5}{8} m_{R}^{2}-\frac{c_{d}}{F}\left(\frac{24 c_{d}}{5 F}-\frac{4 c_{m}}{F}\right) M_{K}^{2}-\frac{3 c_{d}^{2}}{m_{R}^{2} F^{2}}\left(1-\frac{24 c_{d}^{2}}{5 F^{2}}\right)\left(\frac{128 \pi F^{2}}{5}\right)^{2}\right. \\
& \left.-i \frac{c_{d}}{F}\left[3 \frac{c_{d}}{F}-\left(\frac{3 c_{d}}{5 F}-\frac{4 c_{m}}{F}\right) \frac{M_{K}^{2}}{m_{R}^{2}}-\frac{3 c_{d}}{F}\left(1-\frac{24 c_{d}^{2}}{5 F^{2}}\right)\left(\frac{128 \pi F^{2}}{5 m_{R}^{2}}\right)^{2}\right] \frac{128 \pi F^{2}}{5}\right\}, \\
s_{-}= & \frac{7}{5} M_{K}^{2}+\frac{24 m_{R}^{2} c_{d}^{2}}{5 F^{2}}\left(\frac{128 \pi F^{2}}{5 m_{R}^{2}}\right)^{2}-i\left[1-\frac{24 c_{d}^{2}}{5 F^{2}}\left(\frac{128 \pi F^{2}}{5 m_{R}^{2}}\right)^{2}\right] \frac{128 \pi F^{2}}{5} .
\end{aligned}
$$

The graphic 1, shows that the inclusion of the pion mass is not numerically important and off-shell
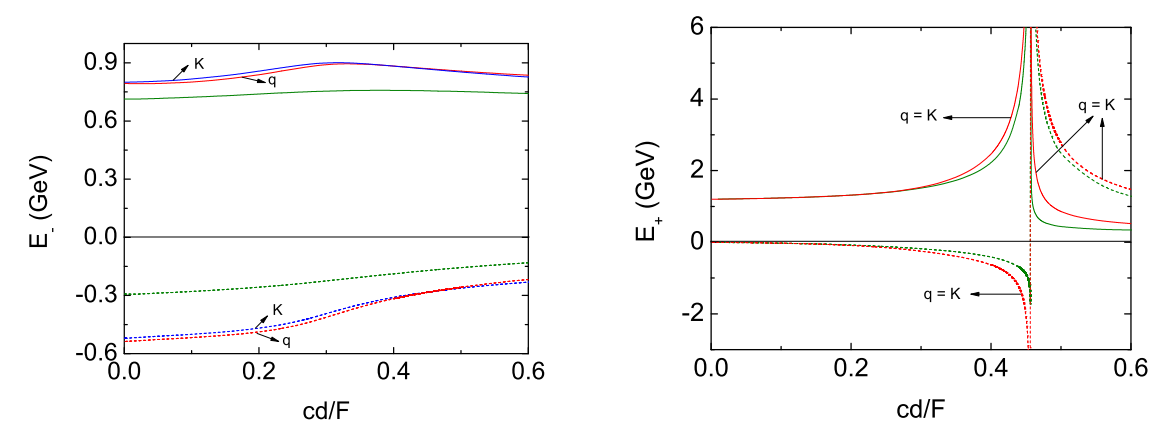

Figure 1: Real (full) and imaginary (dashed) components of respectively functions $E_{-}=\sqrt{s_{-}}$and $E_{+}=\sqrt{s_{+}} \quad$. Green $\equiv$ numerical; $K$ and $q$ are K-matrix and quadratic approximation.

effects in the two-meson propagator do influence the positions of the poles. We recognize $\sqrt{s_{+}}$as been $K_{0}^{*}(1430)$ and $\sqrt{s_{-}}$as been $\kappa$.

The following scenario is supported by eqs.(9-10):

- if resonance $R$ is absent we have only $\sqrt{s_{-}}$originated in contact interaction;

- if resonance $R$ is present and $c_{d}=c_{m}=0$, we have a bound state $\sqrt{s_{+}}$in the real axis at $s=m_{R}^{2}$;

- if $c_{d} \neq 0$ the mass and width of $\sqrt{s_{+}}$, eq.(9), increase monotonically, driven by the factor $A$ in the denominator;

- the pole $\sqrt{s_{+}}$blows up at the critical value $c_{d} / F=\sqrt{5 / 24} \rightarrow$ beyond this point, just $\sqrt{s_{-}}$is present.

Prediction: $K_{0}^{*}(1430)$ pole at $[(1.414 \pm 0.006)-i(0.145 \pm 0.010)] \mathrm{GeV} \Rightarrow m_{R}=1.1865 \pm 0.079$ $\mathrm{GeV}$ and $c_{d}=0.02786 \pm 0.00078 \mathrm{GeV}$

$\Rightarrow \kappa$ pole at $(0.7505 \pm 0.0010)-i(0.2363 \pm 0.0023) \mathrm{GeV}$.

\section{References}

[1] G. Colangelo, J. Gasser and H. Leutwyler, Nucl. Phys. B 603 (2001) 125.

[2] A. Pich, E. Rafael, G Ecker, J. Gasser, Nucl. Phys. B 321 (1989) 311.

[3] J.A. Oller and E. Oset, Nucl. Phys. A 620 (1997) 438. 